\title{
Scanning electron microscopic study of a Ciloxan bottle blocked by ciprofloxacin crystals

\begin{abstract}
Purpose To report blockage of a commercially available ciprofloxacin bottle by white crystalline deposits. This study evaluated the ultrastructural features of the ciprofloxacin crystals.

Methods A patient underwent intensive topical treatment of an infectious corneal ulcer with commercially available ciprofloxacin $0.3 \%$ ophthalmic solution. During treatment, the patient was unable to obtain medication from the ciprofloxacin bottle and required a new prescription. Examination of the bottle revealed that about $50 \%$ of the medication remained, but compression of the bottle with any amount of force failed to deliver any medication. On closer examination, a white material partially filled the nozzle track of the bottle and was on the outer bottle near the nozzle and the inner surface of the bottle cap. These white crystalline deposits were evaluated by scanning electron microscopy. Results Plate-like, needle, cable and spaghetti-like crystals were found. The needle crystals formed multiple petaloid patterns. Conclusions This is the first report of blockage of a commercially available ciprofloxacin $0.3 \%$ bottle by ciprofloxacin crystals and the inability to deliver medication from the bottle. Ultrastructural study of the white crystalline deposits revealed four types of ciprofloxacin crystals.
\end{abstract}

\section{T. John}

Department of

Ophthalmology

Loyola University Medical Center

Maywood

IL 60153, USA

Thomas John, MD Chicago Cornea Research Center

7060 Centennial Drive

Tinley Park

IL 60477, USA

Tel: +1 (708) 4292223

Fax: +1 (708) 4292226

e-mail: cornea999@aol.com

Proprietary interest: None
Key words Ciloxan, Ciprofloxacin, Crystals, Scanning electron microscopy

Ciprofloxacin ophthalmic solution is commercially available in a $0.3 \%$ concentration (Ciloxan, Alcon Laboratories, Fort Worth, TX), which is effective against a wide range of ocular pathogens, except fungi and viruses. ${ }^{1}$ Because of the drug's broad-spectrum coverage of ocular pathogens and the ease of using a single, commercially available ophthalmic antibiotic solution compared with a pharmacycompounded, fortified antibiotic solution, ciprofloxacin has increasingly been used to treat infectious keratitis and corneal ulcers.
The adverse effects of ocular ciprofloxacin have been described as mild and not serious. ${ }^{2}$ One side-effect is the formation of a white crystalline precipitate on the superficial portion of the treated cornea. ${ }^{1-5}$

The author previously reported the first description of the ultrastructural features of these white crystalline ciprofloxacin deposits on the cornea and soft contact lens. ${ }^{6,7}$ This is the first report of blockage of a commercially available ciprofloxacin $0.3 \%$ bottle by ciprofloxacin crystals and the ultrastructural characterisation of the white crystals in the bottle.

\section{Materials and methods}

A patient underwent intensive topical treatment of an infectious corneal ulcer with commercially available ciprofloxacin $0.3 \%$ eye drops for a total period of about 6 weeks. During treatment, dense white ciprofloxacin crystalline deposits formed in the nozzle tract of the bottle, the outer surface of the drug delivery side of the bottle, and on the inner surface of the bottle cap (Figs. $1,2)$ after the bottle had been open for about 3 weeks. The bottle was stored at room temperature. The bottle cap was replaced between periods of use. About $50 \%$ of the original drug solution remained in the bottle, but the patient was unable to use it because the deposits blocked the neck of the bottle.

Mechanical compression of the bottle failed to release the blockage. These crystalline deposits were studied using scanning electron microscopy (SEM).

\section{SEM}

The specimen was mounted on an aluminium SEM stub with double-sided adhesive tape, sputter-coated with $20 \mathrm{~nm}$ of gold, and viewed under Zeiss DSM-940 and Cambridge Stereoscan-120 scanning electron microscopes. 


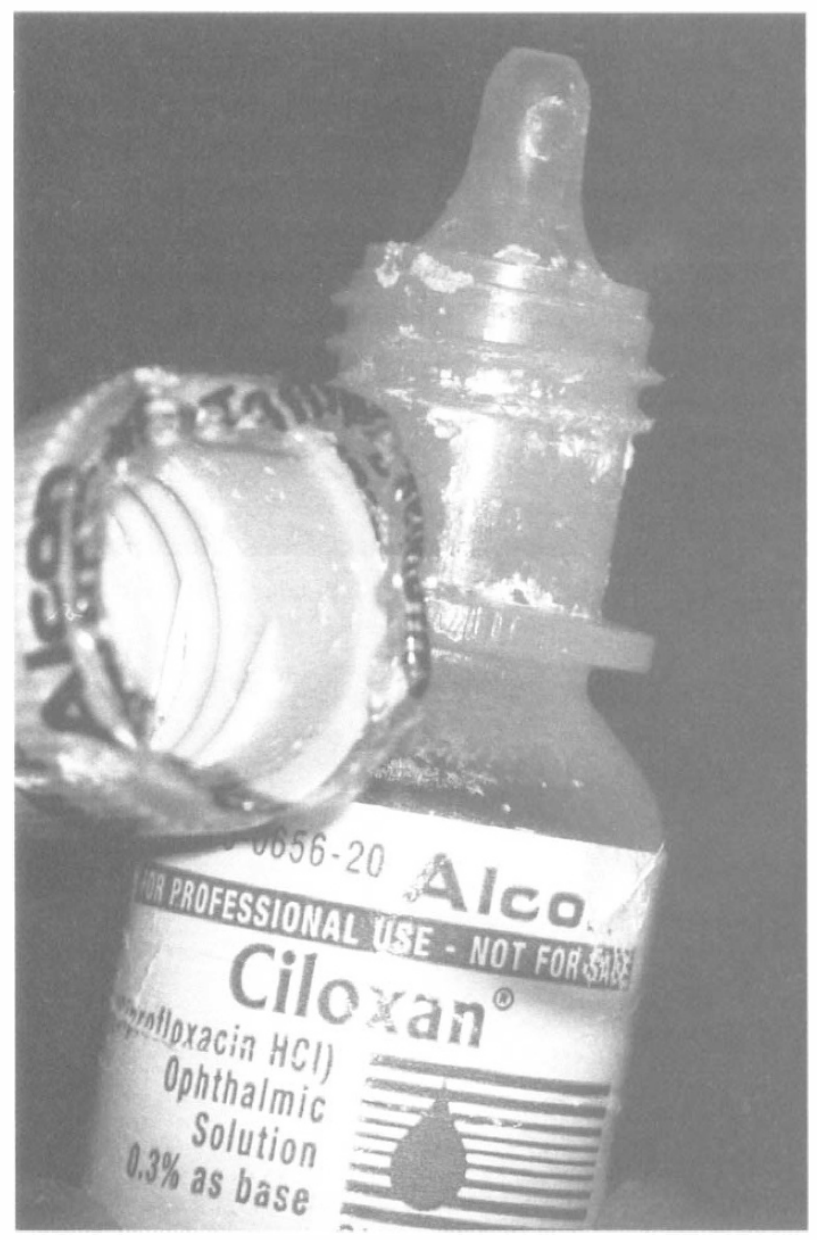

Fig. 1. Ciprofloxacin bottle with white crystalline deposits on the outer surface of the bottle and the inner surface of the bottle cap.

\section{Results}

Four types of crystals were detected by SEM: plate-like crystals (Fig. 3), needle crystals, cable crystals (Fig. 4) and spaghetti-like crystals (Fig. 5). Fig. 6 displays the four types of crystals seen in this scanning electron microscopic study.

The plate-like crystals were flat with a smooth surface and appeared to form a sheet-like pattern. The needle crystals, which formed multiple petaloid patterns, were shorter compared with the other types of crystals.

The descending order of thickness of the crystals was as follows: plate-like crystals, cable crystals, needle crystals and spaghetti-like crystals. The plate-like crystals, cable crystals and needle crystals were straight, while the spaghetti-like crystals formed multiple curvilinear patterns. Morphologically similar crystals seemed to interdigitate among themselves.

The crystals with a similar morphological pattern seemed to group together. In some areas, a single type of crystal was seen; in other areas a combination of the different crystalline forms was seen.

\section{Discussion}

Ciprofloxacin $0.3 \%$ solution is the first of the fluoroquinolone antibacterial agents that was approved for ocular use in the United States. Before this, fortified

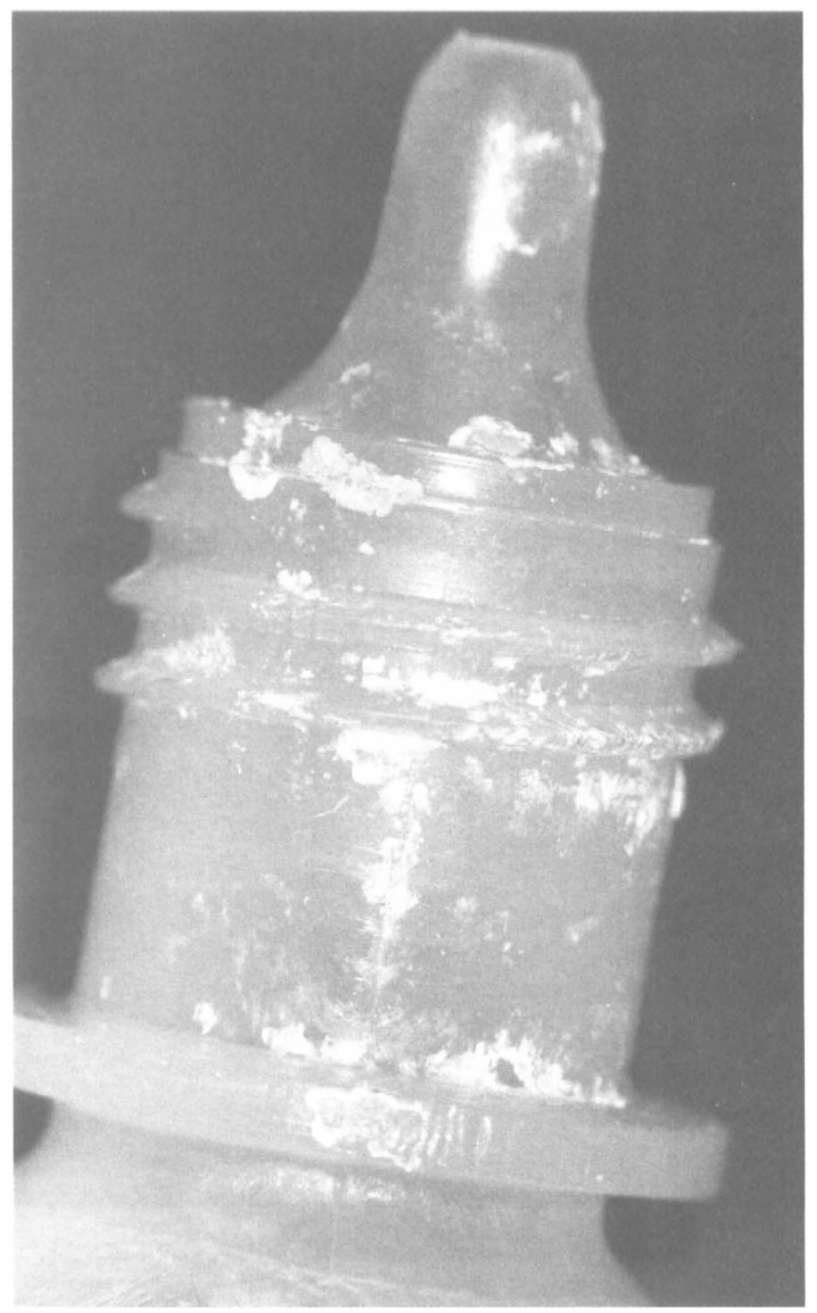

Fig. 2. Magnification of the ciprofloxacin bottle showing the white crystalline deposits on the outer and inner areas of the drug delivery side of the bottle.

antibiotic solutions were formulated in the pharmacy and used to treat corneal ulcers. The ease of availability of ciprofloxacin and its broad-spectrum coverage of bacterial pathogens led to its increasing use to treat infectious keratitis and corneal ulceration. Ciprofloxacin continues to be used in place of standard, combinationfortified antibiotics that have a limited shelf life.

The potential side-effects of topical ciprofloxacin include formation of white crystalline deposits on the superficial cornea and the eye lashes, discomfort, foreign-body sensation, chemosis, punctate epithelial erosion, eyelid oedema, conjunctival hyperaemia, tearing, itching and superficial punctate keratitis. ${ }^{2}$ The most common ocular side-effect associated with ciprofloxacin therapy is the formation of a white crystalline precipitate on the corneal surface. ${ }^{2,3}$ In a clinical study of 148 culture-proven cases of bacterial keratitis treated with ciprofloxacin $0.3 \%$ solution, 35 patients developed a white crystalline precipitate on the corneal surface. $^{2}$ In 25 of these patients, the precipitate was located in the area of the corneal ulceration. ${ }^{2}$

The author first reported the scanning and transmission electron microscopic features of these white crystalline deposits on the cornea and soft contact lens. ${ }^{7}$ 


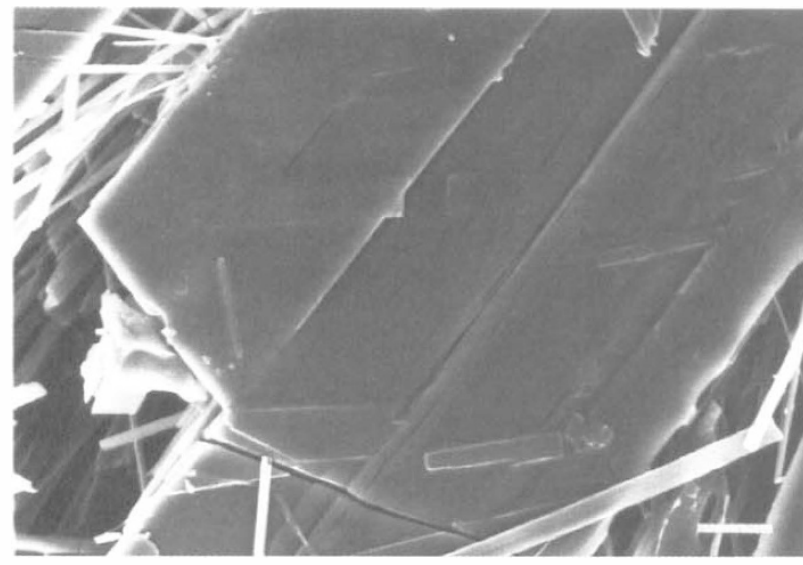

Fig. 3. Scanning electron micrograph of ciprofloxacin deposits showing large plate-like crystals. Scale bar represents $10 \mu \mathrm{m}$.

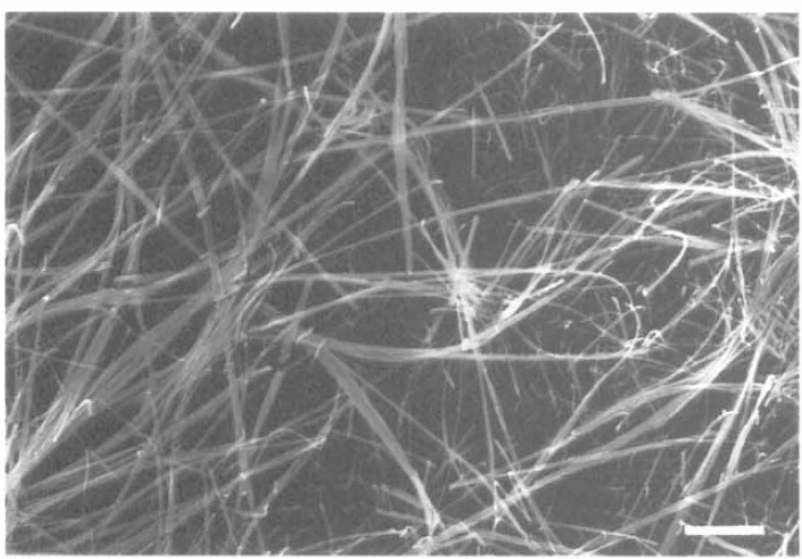

Fig. 5. Scanning electron micrograph displaying spaghetti-like crystals of ciprofloxacin. Scale bar represents $10 \mu \mathrm{m}$.

The ultrastructural study demonstrated the presence of plate-like crystals on the cornea and soft contact lenses. ${ }^{6,7}$ In addition, three other types of crystals, namely needle, cable and spaghetti-like, were observed in the present study. The exact mechanism for the formation of these white crystalline deposits is not fully understood. The bottle cap had the plastic security seal still attached to the cap (Fig. 1), and this may have prevented complete closure of the bottle cap and consequent exclusion of air. This may have contributed to evaporation of the solution and allowed deposits to form within the nozzle of the bottle and in the bottle cap. It is important for the patient to remove the plastic security seal and discard it prior to use of the medication. It is also equally important to replace the cap firmly after each use, follow the manufacturer's recommendation regarding storage of the medication between use, and possibly to discard the drops about 28 days after opening the seal of the medication bottle.

Blockage of the ciprofloxacin bottle nozzle by these crystals prevents use of the medication, and could not be resolved by mechanical compression of the bottle.

This is the first report of blockage of a commercially available ciprofloxacin $0.3 \%$ bottle by crystals and the inability to deliver any medication from the bottle. Ultrastructural study of these white crystalline deposits revealed four types of ciprofloxacin crystals.

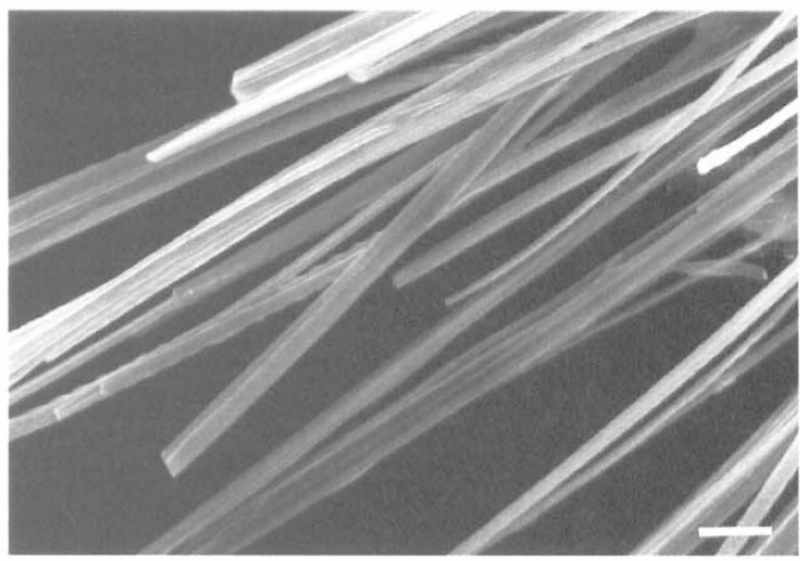

Fig. 4. Scanning electron micrograph displaying cable crystals of ciprofloxacin. Scale bar represents $10 \mu \mathrm{m}$.

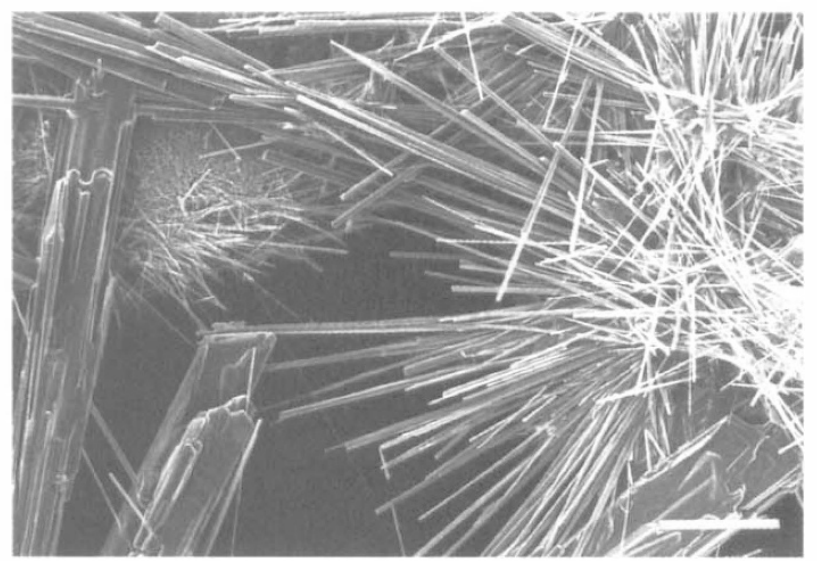

Fig. 6. Four types of ciprofloxacin crystals are seen: plate-like crystals (lower left), needle crystals (upper right), cable crystals (lower right) and spaghetti-like crystals (upper left). Scale bar represents $100 \mu \mathrm{m}$.

Supported by the Loyola Cornea Research Fund and Richard A. Perritt Charitable Foundation.

\section{References}

1. Neu HC. Microbiological aspects of fluoroquinolones. Am J Ophthalmol 1991;112:S15-24.

2. Leibowitz HM. Clinical evaluation of ciprofloxacin $0.3 \%$ ophthalmic solution for treatment of bacterial keratitis. Am J Ophthalmol 1991;112:S34-47.

3. Wilhelmus KR, Hyndiuk RA, Caldwell DR, Abshire RL, Folkens AT, Godio LB, for the ciprofloxacin ointment/ bacterial keratitis study group. 3\% ciprofloxacin ophthalmic ointment in the treatment of bacterial keratitis. Arch Ophthalmol 1993;111:1210-8.

4. Insler MS, Fish LA, Silbernagel J, Hobden JA, O'Callaghan RJ, Hill JM. Successful treatment of methicillin-resistant Staphylococcus aureus keratitis with topical ciprofloxacin. Ophthalmology 1991;98:1690-2.

5. Kanellopoulos AJ, Miller F, Wittpenn JR. Deposition of topical ciprofloxacin to prevent re-epithelialization of a corneal defect. Am J Ophthalmol 1994;117:258-9.

6. John T. Ultrastructural characteristics of corneal deposits of ciprofloxacin. Ophthalmology 1994;(Suppl)101:104.

7. John T. Ciprofloxacin deposits on the cornea and soft contact lens: an ultrastructural study. Invest Ophthalmol Vis Sci 1995;36(Suppl):S152. 